\title{
A ribosomal function is necessary for efficient splicing of the T4 phage thymidylate synthase intron in vivo
}

\author{
Katharina Semrad and Renée Schroeder ${ }^{1}$ \\ Institute of Microbiology and Genetics, Vienna Biocenter, 1030 Wien, Austria
}

\begin{abstract}
Splicing of the group I intron of the T4 thymidylate synthase (td) gene was uncoupled from translation by introducing stop codons in the upstream exon. This resulted in severe splicing deficiency in vivo. Overexpression of a UGA suppressor tRNA partially rescued splicing suggesting that this in vitro self-splicing intron requires translation for splicing in vivo. Inhibition of translation by the antibiotics chloramphenicol and spectinomycin also resulted in splicing deficiency. Ribosomal protein S12, a protein with RNA chaperone activity, and CYT-18, a protein that stabilizes the three-dimensional structure of group I introns, efficiently rescued the stop codon mutants. We identified a region in the upstream exon that interferes with splicing. Point mutations in this region efficiently alleviate the effect of a nonsense codon. We infer from these results that the ribosome acts as an RNA chaperone to facilitate proper folding of the intron.
\end{abstract}

[Key Words: Group I intron splicing; translation; RN A chaperones]

Received October 13, 1997; revised version accepted January 29, 1998.

Group I introns occur in ribosomal RNA, tRNA, and in protein encoding genes. Many of these introns are selfsplicing in vitro and do not require proteins for activity (Cech 1990). Group I introns fold into a compact defined structure, embedding the $5^{\prime}$ splice-site into a stem (P1), which is docked into the catalytic core of the molecule (Waring et al. 1986; M ichel and Westhof 1990; Cech et al. 1994). For group I introns that disrupt rRNAs and tRN As, it was shown that the exon structure influences folding of P1, and as a consequence, splicing efficiency (Woodson and Cech 1991; Woodson 1992; Woodson and Emerick 1993; Zaug et al. 1993). Exon sequences surrounding the splice sites of pre-mRNAs, however, should be mainly unstructured to ensure efficient translation. M ost group I mRNA introns reside in prokaryotes or in eukaryotic organelles where transcription, splicing and translation are linked events. The ribosome might reach a $5^{\prime}$ splice site before the $3^{\prime}$ splice site is even transcribed. This situation clearly requires tight regulation to prevent aberrant translation products. Additionally, many group I introns encode proteins in-frame with the preceding exons, or, as in the T4 phage introns, expressed independently from their own promoters located within the intron (Lazowska et al. 1980; Gott et al . 1988).

RN A catalysis depends on complex tertiary structures. In vivo, many group I introns require splicing factors to promote the correct folding into the catalytically active tertiary structure (Lambowitz and Belfort 1993). In vitro, splicing can occur in the absence of protein factors, but activity is most often limited by incorrectly folded RN A

${ }^{1}$ Corresponding author.

E-MAIL renee@gem.univievieac.at; FAX 4317986224. molecules (Walstrum and Uhlenbeck 1990). Two proteins that promote the folding of group I introns, have been extensively studied: the tyrosyl-tRNA synthetase of Neurospora crassa (CYT-18) (Lambowitz and Perlman 1990) and the CBP2 protein from yeast mitochondria (Gampel et al. 1989).

CYT-18 is involved both in charging its cognate tRN A and in promoting splicing of group I introns. CYT-18 is also capable of binding group I introns from other species, like the T 4 phage thymidylate synthase $(\mathrm{td})$ and the Tetrahymena thermophila LSU introns. In the latter case, CYT-18 does not bind to the full-size intron, rather, it functionally replaces the P5abc domain, stabilizing the P4-P6 domain (Murphy and Cech 1993; Mohr et al. 1994). CYT-18 binds to group I introns by first contacting the P4-P6 domain to form a scaffol d for the assembly of the P3-P9 domain. CYT-18 interacts with both helical domains of the intron on the side opposite to the active site cleft, which accommodates the 5' splice-site by recognizing the shape of the molecule through contacts with the phosphate backbone (Caprara et al. 1996b). CYT-18 seems not to contact the P1 stem harboring the splice site. It restores splicing in vitro for splicing-defective mutations throughout the intron, except those located in the P1 and P2 stems of the T4 phage td intron (Mohr et al. 1992). In contrast, the CBP2 protein, which facilitates folding of the bl 5 intron of yeast mitochondria, does this by assembling the intron core with the P1 domain containing the splice site (Weeks and Cech 1995a,b).

RNA folding encounters two problems: It easily be comes kinetically trapped in inactive conformations and its three dimensional structure is often thermodynami- 
cally misfavored over alternative folds. RNA-binding proteins that help overcome these problems by preventing misfolding or by resolving misfolded species are defined as RNA chaperones (Herschlag 1995). While the CYT-18 and CBP2 proteins stabilize a specific RNA structure, several other RNA-binding proteins, such as the nucleocapsi d protein of HIV and the hnRNP A 1, have been assigned a nonspecific RN A-binding activity. These proteins accelerate ribozyme catalysis in a more general way, and once the RNA is folded, they are no longer needed for activity (Herschlag 1995). Similarly, several ribosomal proteins of Escherichia coli, especially S12, were found to stimulate trans-splicing of the T4 phage td intron in vitro. S12 does not bind strongly or specifically to the intron RN A, but has a rather broad specificity for RN A. Like other RN A chaperones, it facilitates catal ysis of the hammerhead ribozyme (Tsuchihashi et al. 1993; Herschlag et al. 1994). S12 was suggested to have RN A chaperone activity (Coetzee et al. 1994).

Aminoglycoside antibiotics, which affect translation accuracy, have been shown to inhibit splicing of group I introns in vitro (von Ahsen et al. 1991). To study the effect of these antibiotics on splicing in vivo, we uncoupled splicing from translation by introducing a nonsense codon in the upstream exon of the td gene. Surprisingly, even in the absence of antibiotics, this stop codon, located 223 nucleotides upstream of the 5' splice site, reduced in vivo splicing to an undetectable level. Further analysis of this phenomenon revealed that the ribosome plays an essential role in splicing of this mRN A intron in vivo.

\section{Results}

Stop codons in the upstream exon of the T4 phage td gene lead to splicing deficiency in vivo

Stop codons were introduced into exon 1 of the td gene at various positions by site-directed mutagenesis (Fig. 1A). In vivo, splicing in the mutants containing stop codons was tested via a primer-extension assay (Fig. 1B). Total cellular RNA of E. coli strains transformed with plasmids harboring the T 4 td gene was isolated, and the efficiency of splicing was tested by reverse transcription with a primer (NBS2) that hybridizes at the $5^{\prime}$ end of exon 2. In the presence of ddTTP, dATP, dCTP, and dGTP, primer extension stops at the first $A$ of the template after ddTTP is incorporated. Extension of the premRN A results in a product that is 5 nucleotides Ionger than the primer $(P+5)$, while the mature mRN A allows the addition of 16 nucleotides (Fig. $1 \mathrm{C}$ ). To ensure that the NBS2 primer does not hybridize to endogenous E. coli sequences, a strain without the td gene was tested (Fig. 1C, lane 1). As a splicing-deficient control, a td mutant with a mutation in the catalytic core was used (td C870U, Fig. 1C, lane 2; Schroeder et al. 1991). In addition to the mature splicing product, a cryptic splice site is utilized 29 nucleotides upstream of the $5^{\prime}$ splice site, resulting in a $\mathrm{P}+8$ extension product (Chandry and Bel-
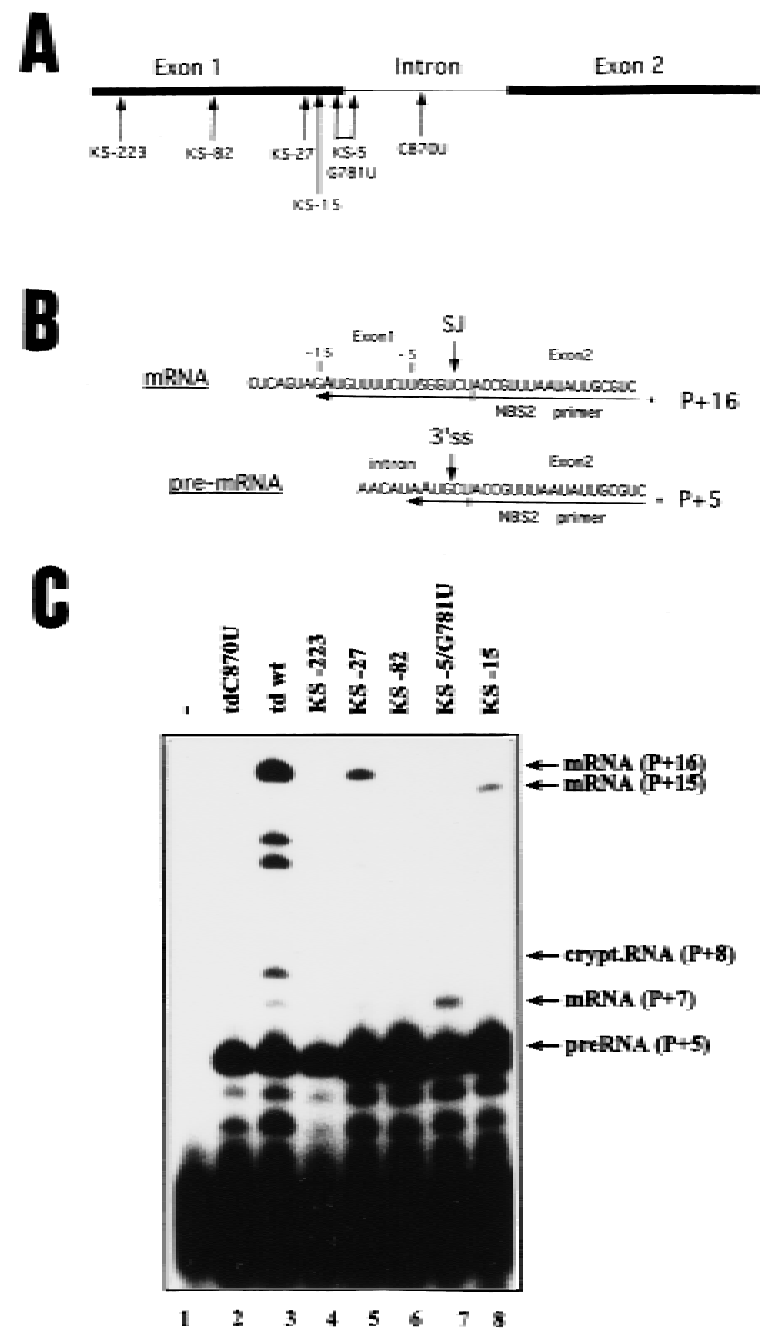

Figure 1. In vivo splicing of the stop codon mutants. (A) Position of the stop codons introduced into exon 1 of the T4 phage td gene and of the $\mathrm{C} 870 \mathrm{U}$ intron mutant. N umbering of nucleotides indicates the distance to the $5^{\prime}$ splice site. For exact sequence of mutations see Table 1. (Solid bars) Exons; (thin lines) intron sequences. (B) Schematic representation of the primerextension assay for splicing in E. coli. Extension of the ${ }^{32} \mathrm{P}$ labeled NBS2 primer, which hybridizes to exon 2 sequences, stops at the first $A$ of the template in the presence of $d A T P$, dCTP, dGTP and high concentrations of ddTTP. $(P+x)$ Length of the extension product. pre-RN A is extended by 5 nucleotides $(P+5)$ and mRNA is extended by 16 nucleotides $(P+16)$. (SJ) Spliced junction; (3' ss) 3' splice site. (C) Primer-extension assay for splicing. The RNAs were isolated from cells containing no td gene (lane 1), the splicing-deficient mutant (td C870U, Iane 2 ), the wild-type gene (lane 3 ), or the stop codon mutants as indicated (lanes 4-8). For the stop codon mutants KS-15 and $\mathrm{KS}-5 / \mathrm{G} 781 \mathrm{U}$ the reverse transcripts of the mRNA differ in length because of the $U$ to $A$ mutation at position -15 or at position -5 and result in $\mathrm{P}+15$ and $\mathrm{P}+7$ extension products, respectively.

fort 1987). Bands at positions $P+12 / 13$ (Fig. 1C, lane 3) are unidentified products, which may result from a premature stop of reverse transcription. The gel was strongly overexposed during autoradiography to detect 
Table 1. Effect of nonsense codons in exon 1 on splicing in vivo

\begin{tabular}{llc}
\hline Mutation & Designation & Percent mRN A \\
\hline Wild type & Wild-type td & $41 \pm 1.6$ \\
T546A; G547T & KS-223 & $<1$ \\
T687A & KS-82 & $<1$ \\
C742T; A743G & KS-27 & $10 \pm 1.9$ \\
G754T; T756A & KS-15 & $6.8 \pm 2.2$ \\
T764A; G781T & KS-5/G781U & $15.4 \pm 4.1$ \\
C870T & td C870U & $<1$ \\
\hline
\end{tabular}

Quantification of primer extension products. Radioactivity was determined on a Phosphorlmager, and splicing activity is given in percent mRNA calculated from (mRNA + cryptic RNA) $\times$ 100/(pre-RNA + mRNA + cryptic RNA).

low splicing activities. As can be seen in Figure $1 \mathrm{C}$ and Table 1, splicing activities in the stop codon mutants are significantly lower than in the wild type, depending on the position of the stop codon. In the mutants, where translation stops 223 and 82 nucleoti des upstream of the $5^{\prime}$ splice site, splicing activity is bel ow $1 \%$, whereas the wild-type construct splices to $40 \%$. The closer the stop codon is located to the $5^{\prime}$ splice site, the higher is the splicing activity (Table 1). Stop codons at positions -27 , -15 , and -5 show $10 \%, 7 \%$, and $15 \%$ splicing, respectively. Mutants KS-5/G781U and KS-15 have altered mRN $A$ sequences and reveal products $P+15$ and $P+7$ caused by a prior $A$ appearing in the sequence (Fig. 1C, lanes 7,8$)$. Even when the stop codon is only two codons distant to the $5^{\prime}$ splice site, splicing activity is significantly reduced.

The stop codon mutations do not affect in vitro splicing activity. RNA of the wild type and the stop codon mutants was obtained by in vitro transcription and spliced in vitro as described in Materials and Methods. Splicing activities of the wild type and the stop codon mutants were indistinguishable, showing that the effect observed in vivo is not attributable to a splicing deficiency of the intron itself (data not shown).

\section{Stability of the mRNA is not affected by the stop codons}

It was shown that stop codons close to the $5^{\prime}$ end of mRNAs in Bacillus subtilis induce mRNA degradation in vivo ( $\mathrm{N}$ ilsson et al. 1987). To test whether the effect was attributable to a destabilization of mRNA, an intronless version of the td gene was constructed (td $\Delta$ intron) and a UGA codon was introduced at position -82 (td $\Delta$ intron/KS-82). Cells were grown to an $\mathrm{OD}_{600}$ of 0.2 , then transcription was inhibited by the addition of rifampicin. Time points were taken up to $40 \mathrm{~min}$, and mRNA amounts were compared via a primer-extension assay for the intronless construct with and without the nonsense codon (Fig. 2). For the primer-extension assay, the same amounts of total cellular RN A were used. No differences in the amounts of td-specific mRN A between both constructs are detectable, indicating that the nonsense codon does not affect mRN A stability and that the effect seen on splicing is not attributable to a rapid degradation of the spliced $\mathrm{mRN} A$.

The protein encoded by exon 1 is not required for splicing

Translation of the unspliced pre-mRNA is terminated at the $5^{\prime}$ splice site, because a UAA stop codon is at the very beginning of the intron, and results in a protein product cal led N H2-TS (Belfort et al. 1987). Introducing stop codons in the upstream exon prevents synthesis of this protein. To test whether the translation product of exon 1 is essential for splicing, we extensively al tered its amino acid sequence by del eting nucl eotide -182 of exon 1 , causing a frameshift, and by inserting a C $3^{\prime}$ of G-18,

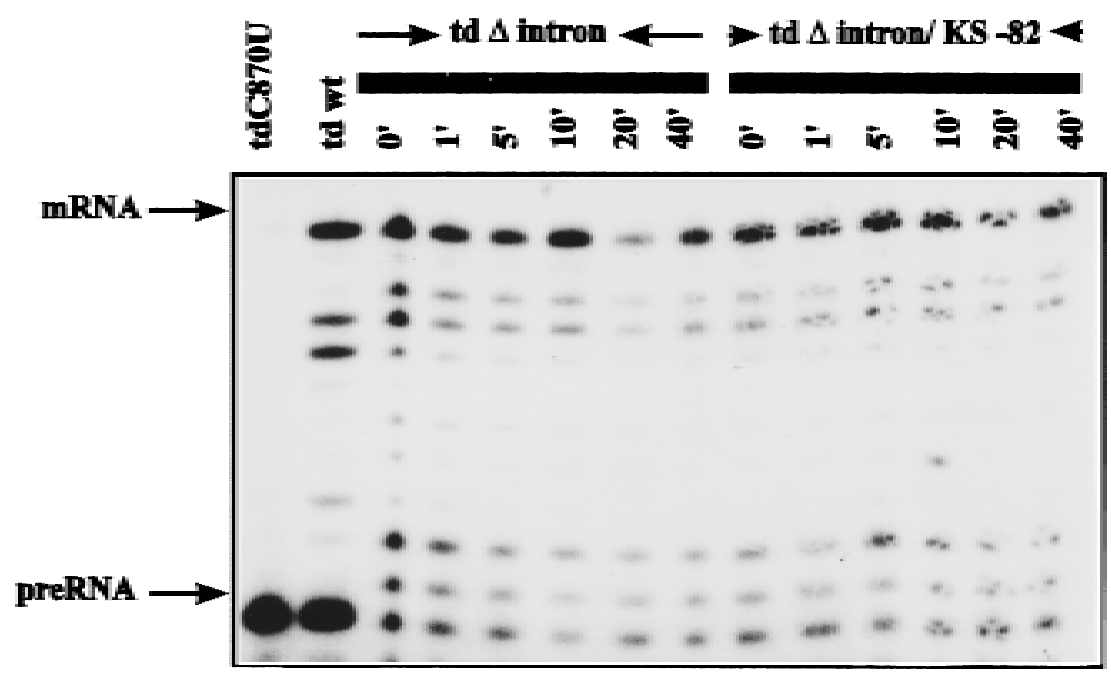

1
34
7
8910 $\begin{array}{llll}11 & 12 & 13 & 14\end{array}$
Figure 2. Stability of a nonsense codon containing mRNA. Comparison of the mRNA stability of the intronless mutants without and with a stop codon at position -82 . The intronless constructs were grown to exponential phase and samples were taken before the addition of rifampicin $\left(0^{\prime}\right)$ and after its addition ( $1^{\prime}$ to $\left.40^{\prime}\right)$. mRN A amounts were assayed via primer-extension assay. (Lane 1 ) C870U mutant; (lane 2) wild type; (lanes 3-8) intronless td gene; (lanes 9-14) intronless td gene with stop codon at position -82 . 
A
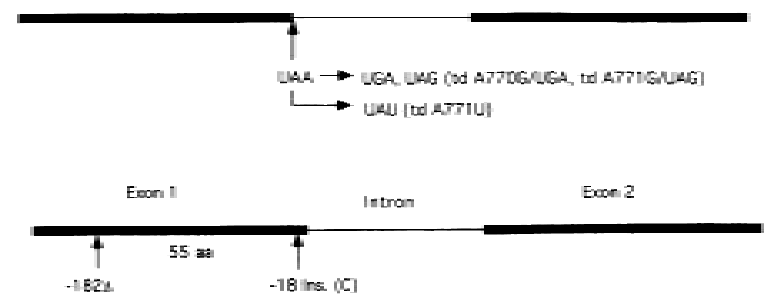

B

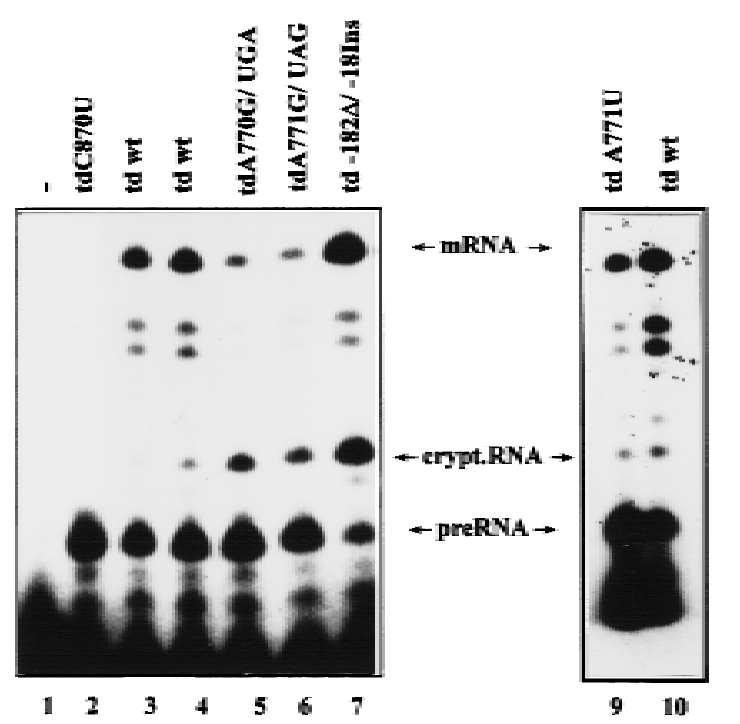

C
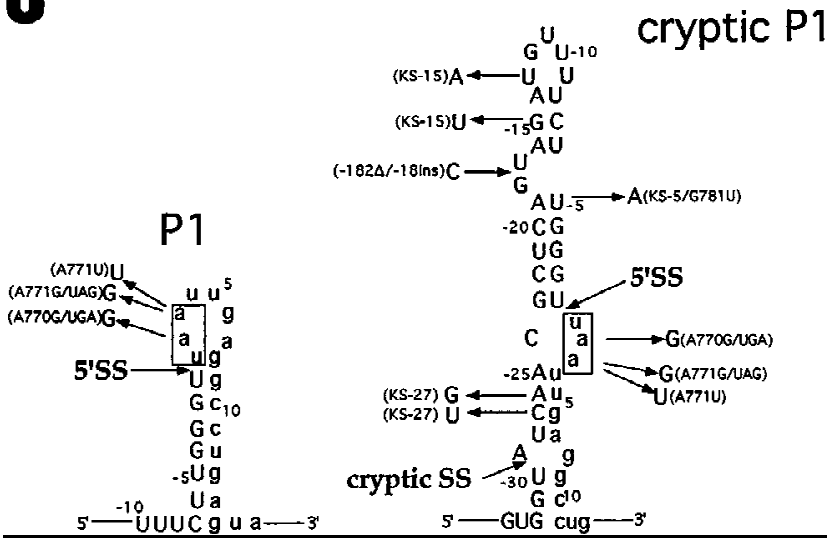

Figure 3. Splicing of td mutants affecting the UAA stop codon at the 5 ' site and the reading frame of exon 1 . (A) The UAA stop codon, which immediately follows the 5 ' splice site was mutated to UGA and UAG or readthrough (tdA 771U). The amino acid sequence of the exon 1 product was modified via deletion of nucleotide -182 and insertion of a $C$ at position -18 , resulting in a product with 55 altered amino acids. (B) Splicing activity. The RNAs were isolated from E. coli cells containing no td gene (lane 1 ), the mutant tdC870U (lane 2), the wild type (lane 3) or the mutants described (lanes 5-9). The bands referring to mRN A, cryptic RN A and precursor are indicated by arrows. (C) P1 and cryptic P1. (P1) Sequences around the $5^{\prime}$ splice site (5' ss) pair with intron sequences forming the P1 stem. Exon sequences are in uppercase, intron sequences in lowercase letters. The UAA stop codon following the $5^{\prime}$ ss is boxed. Positions al tering this stop codon are indicated by arrows and the exchanged nucleotide is indicated. (Cryptic P1) An al ternative fol ding of sequences surrounding the $5^{\prime}$ splice site is shown (Chandry and Belfort 1987). The original and the cryptic splice sites are indicated by arrows, as well as positions al tered by mutations that stabilize the cryptic P1 folding. N egative numbers indicate distances to the $5^{\prime}$ splice site, positive numbers are intron positions.

restoring the original reading frame before the $5^{\prime}$ splice site (Fig. 3A). This results in a protein with an altered sequence over 55 amino acids, most probably disrupting its function, as this part of the protein is highly conserved between species (Carreras and Santi 1995). In vivo splicing activity of this construct was tested and found to be twice as high as for the wild type; in addition, the cryptic splice product $(P+8)$ was al so strongly enhanced (Fig. 3B, lane 7). The fact that splicing takes place in the absence of an active thymidylate synthase (TS) suggests that the protein product of exon 1 is not essential for splicing. However, it remains possible that some portion of the polypeptide could be required. The fact that both splicing and cryptic splicing are enhanced as compared to the wild type suggests further that this protein regulates expression of the gene. It is known that human and
E. coli TS interact with their own mRNAs, repressing translation (Chu et al. 1993; Voeller et al. 1995; Chu and Allegra 1996). It is unknown whether this regulation of translation also occurs in the T4 phage TS. The role of the TS on its own expression needs further investigation.

\section{Changing the natural UAA stop codon in P1 to UGA} or $U A G$ results in less efficient splicing

Like most group I introns in mRN As, the td intron has a stop codon at its $5^{\prime}$ end that is in-frame with the exon. To test whether the nature of this stop codon (UAA) is essential or whether other stop codons could substitute, we changed the original UAA nonsense codon to UGA and UAG. The stop codon was also altered to a sense codon (UAU) by changing A-771 to $U$ (Fig. 3, A and B, 
Table 2. Effects of different nonsense codons and deletion of the UAA stop codon at the $5^{\prime}$ splice site and of altered protein sequence on splicing in vivo

\begin{tabular}{llcr}
\hline Mutation & \multicolumn{1}{c}{ Designation } & $\begin{array}{c}\text { Percent } \\
\text { splicing }\end{array}$ & $\begin{array}{c}\text { Percent } \\
\text { cryptic } \\
\text { RN A }\end{array}$ \\
\hline Wild type & wild type td & $41 \pm 1.6$ & $5.8 \pm 0.7$ \\
A770G & td A770G/UGA & $26.1 \pm-3.3$ & $10 \pm-1.7$ \\
A771G & td A771G/UAG & $29.4 \pm-3.1$ & $12.3 \pm-2.6$ \\
A771T & td A771U & $23.9 \pm-1.7$ & $3.6 \pm-0.2$ \\
$\Delta$ T587, Ins C751 & td $182 \Delta /-18$ Ins & $82.9 \pm-3$ & $23.3 \pm-3.7$ \\
C870T & td C870U & $<1$ & $<1$ \\
\hline
\end{tabular}

Values for the spliced fraction of mRNA + cryptic RNA were determined as in Table 1.

Iane 8). Splicing activities were measured via a primerextension assay and were quantitated by Phosphorlmager measurements (T able 2). The stop codon in L1 just following the $5^{\prime}$ splice site is important, although not essential. In the construct that has the open reading frame into the intron, splicing is down to half of wildtype splicing. The ribosome probably disrupts the intron core structure, as the open reading frame reaches far into the core (P7.2). This confirms the observation made in the T4 nrdB intron, where the elimination of the stop codon also reduced splicing activity (Öhman-Heden et al. 1993).

Splicing is reduced in mutants with the UAG or UGA stop codons replacing the natural UAA stop codon in the intron. While mRNA splicing is significantly decreased, the amount of cryptic splice product is higher compared to the wild type (Fig. 3B, lanes 5, 6 and Table 2). The increase in cryptic splicing is likely attributable to stabilization of the cryptic P1 structure shown in Figure 3C. The al tered $A \rightarrow G$ mutations at positions 770 and 771 enable $G$ to base-pair with $C$ at position -25 . Total splicing, mRNA and cryptic RNA, in the UGA and UAG mutants is reduced to $2 / 3$ of wild-type splicing activity.

\section{Translation inhibitors efficiently inhibit splicing} activity

To illuminate the necessity of translation for efficient splicing activity, we tested the effect of translation inhibitors on splicing in vivo. Chloramphenicol is known to block el ongation of the translating ribosome and spectinomycin inhibits initiation. The td wild-type construct was grown to the exponential phase and incubated for $60 \mathrm{~min}$ with different concentrations $(0,8,40,80$ and $123 \mu \mathrm{M}$ ) of the respective antibiotic. In Figure 4, lanes 1 and 6 , shows splicing in the absence of antibiotic. With increasing amounts of the antibiotic chloramphenicol (lanes 2-5) and spectinomycin (lanes 7-10), splicing decreases dramatically. Both antibiotics are known to affect translation but do not affect splicing in vitro (von A hsen et al. 1992). This demonstrates further the importance of translation for splicing.
A UGA-tRNA suppressor partially rescues splicing of the UGA stop codon mutant

To test whether readthrough of an exon stop codon can rescue splicing, a UGA suppressor tRNA was overexpressed in the KS-82 mutant, which contains a UGA codon in exon 1, 82 nucleotides upstream from the $5^{\prime}$ splice site. The su9 gene coding for a suppressor tRN $\mathrm{A}_{U^{-}}$ $G^{T r p}$ (Kopel owitz et al. 1992) was cotransformed with the mutant KS-82, and splicing was compared to the KS82 mutant strain and to the wild type both without the suppressor (Fig. 5A). The suppressor tRNA slightly rescues splicing. As a tRNA suppressor has to work with low efficiency, otherwise it would be deleterious to the cell, the gel was overexposed to detect very low splicing activity.

The suppressor tRN A was al so tested for its effect on synthesis of TS. The constructs, grown in the E. coli strain C600thy ${ }^{-}$, were tested for growth on thymine-deficient plates (Fig. 5B). The wild-type and the tRN A suppressor strains are the only ones able to grow on minimal medium. This result demonstrates that splicing and translation are rescued when the inserted stop codon is suppressed.

Overexpression of the ribosomal protein S12 slightly raises splicing activity in the stop codon mutants

Recently, it was demonstrated that cellular proteins enhance in vitro trans-splicing of the td intron. They were identified as ribosomal proteins with protein S12 being the most efficient (Coetzee et al. 1994). S12 was proposed to act as an RN A chaperone, which means that it might provide help in correctly folding the ribozyme or in unfolding trapped inactive structures. This protein does not bind specifically to the intron RNA, but rather nonspecifically, with a slight preference for exon sequences. Assuming that ribosomal protein S12 acts as an RN A chaperone in vivo, we probed for the role of the ribosome in

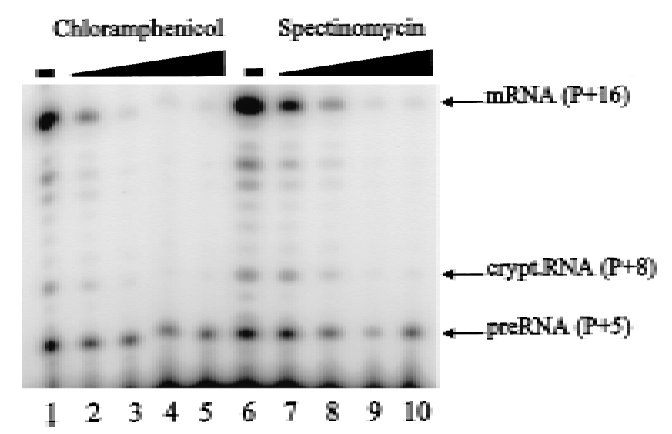

Figure 4. Splicing inhibition by translation inhibitors. Splicing activity was tested in the absence and in the presence of increasing amounts of the antibiotics chloramphenicol and spectinomycin. (Lanes 1,6$)$ In vivo isolated wild-type RN A grown without antibiotic. (Lanes 2-5, 7-10) RNA isolated from the strains grown in the presence of increasing amounts $(8,40,80$ and $123 \mu \mathrm{M}$ ) of chloramphenicol and spectinomycin, respectively. Bands are as indicated in Fig. 1. 
splicing, by overexpressing S12. This might give us a first hint, whether splicing deficiency is attributable to misfolded or unfolded pre-RNA.

The strains of the wild-typetd intron or the stop codon mutants were transformed with a plasmid overexpressing the ribosomal protein S12 (Fig. 6A) (Coetzee et al. 1994). In the wild-type construct, only a slight effect on splicing was observed, and the cryptic spl ice-site product $(P+8)$ was slightly decreased (Fig. 6, lanes 1,2$)$, suggesting that S12 might destabilize the cryptic P1 stem shown in Figure $3 C$. This effect had been observed previously (T. Coetzee and M. Belfort, pers. comm.). S12 slightly raises splicing in all stop codon mutants by a factor of $\sim 2$ (Table 3). The increase in splicing of mutant KS-82 was not quantifiable as splicing in the absence of overexpressed S12 was undetectable. From these results, we conclude that one possible explanation for the splicing deficiency in the stop codon mutants invokes folding problems of the pre-RNA.

The mutant strains, with and without S12 overexpression, were tested for TS synthesis on thymine ${ }^{+}$and thymine ${ }^{-}$selection plates (Fig. 5B). Overexpression of S12 has no effect on translation in the stop codon mutants, indicating that $\mathrm{S} 12$ does not act as a nonsense codon suppressor. S12 overexpression leads to an increase of splicing without affecting translation.
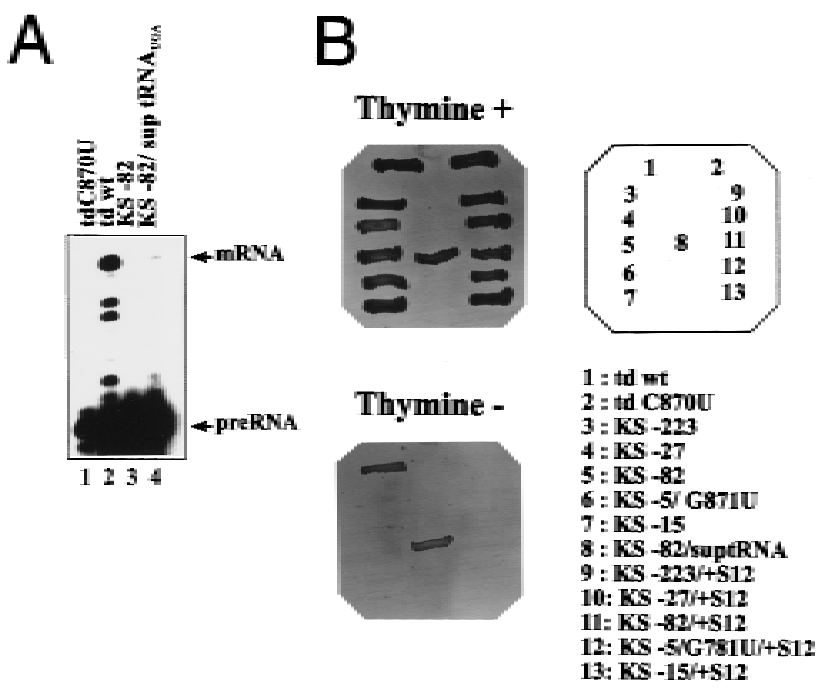

Figure 5. A UGA suppressor tRN A partially rescues splicing in a UGA mutant. Mutant KS-82 was cotransformed with a compatible plasmid encoding a UGA supressor tRNA. (A) In vivo RN A was isolated from the KS -82 mutant alone and from the strain cotransformed with the supressor tRN A on a plasmid, as described in the legend to Fig. 1. As controls the extension products from the wild type and the splicing-deficient mutant (td C870U) are shown. (B) The strains (as indicated) were plated on a thymine-deficient medium to test the suppressor activity and the effect of S12 overexpression on translation. Thy ${ }^{-}$is thymine-deficient medium and $\mathrm{Thy}^{+}$is a full medium.
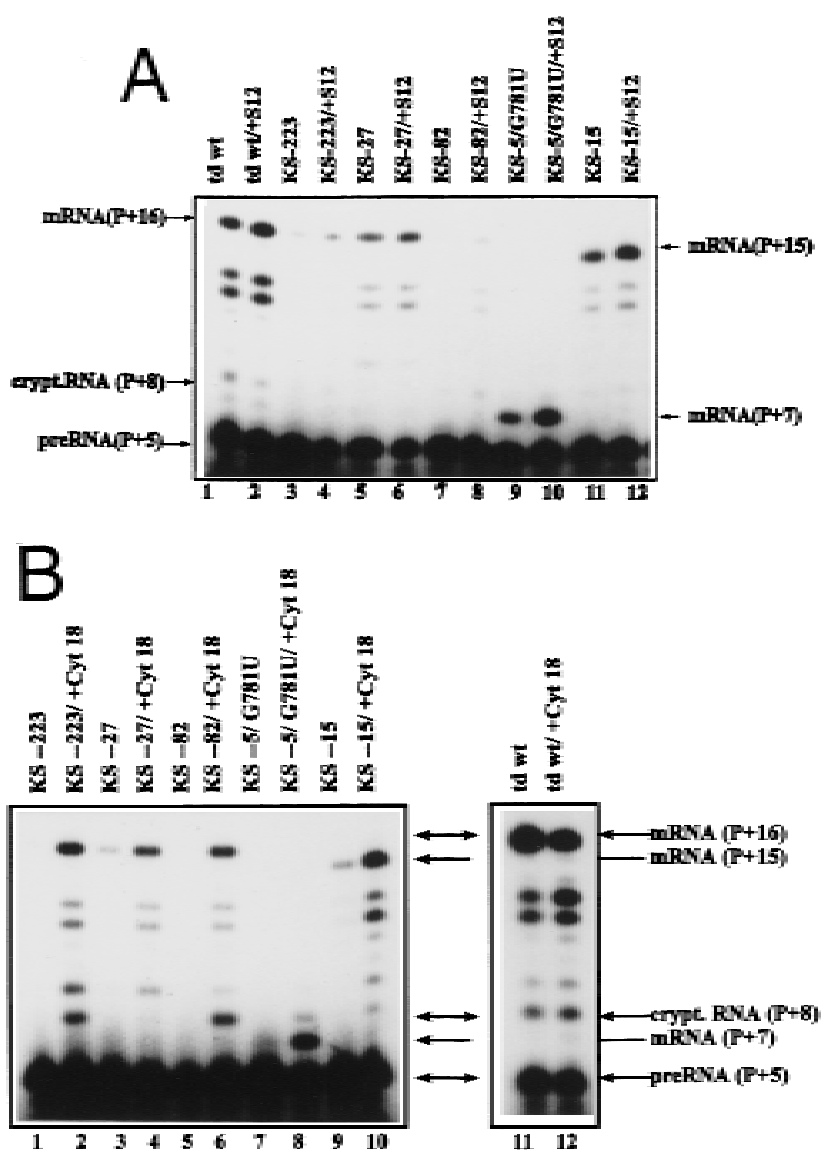

Figure 6. Splicing rescue by ribosomal protein $\mathrm{S} 12$ and by CYT-18. The td wild-type and the stop codon mutants were cotransformed with a compatible plasmid encoding ribosomal protein S12 (A) and CYT 18 (B). In vivo splicing activity was tested via the primer-extension assay. The extension products are labeled as in Fig. 1.

The tyrosyl-tRNA synthetase from N. crassa (CYT-18) efficiently rescues splicing of the stop codon mutants, but aberrant splicing products accumulate

As mentioned before, CYT-18 suppresses structural mutants of the td intron ( $\mathrm{M}$ ohr et al. 1992) and stabilizes the three-dimensional structure of the intron RNA. To test

Table 3. Splicing increase of the stop codon mutants in the presence of overexpressed ribosomal protein S12

\begin{tabular}{lc}
\hline Construct & x-Fold increase in splicing \\
\hline $\mathrm{KS}-223 /+\mathrm{S} 12$ & 2.1 \\
$\mathrm{KS}-27 /+\mathrm{S} 12$ & 1.4 \\
$\mathrm{KS}-82 /+\mathrm{S} 12$ & $>2$ \\
$\mathrm{KS}-5 / \mathrm{G} 781 \mathrm{U} /+\mathrm{S} 12$ & 2.3 \\
$\mathrm{KS}-15 /+\mathrm{S} 12$ & 1.6
\end{tabular}

The values indicate the increase of splicing of the stop codon mutants in the presence of overexpressed ribosomal protein S12 compared to splicing in the absence of overexpressed S12. 
Table 4. Effect of stop codons and compensatory mutations in the $-60 /-80$ region of exon 1 on splicing

\begin{tabular}{llc}
\hline Mutation & Designation & $\begin{array}{c}\text { Relative splicing } \\
\text { activity (\%) }\end{array}$ \\
\hline Wild type & wild-type td & 100 \\
C720A; G718T & KS-51 & 60 \\
G711A; T710G; G709T & KS-60 & 64 \\
T687A & KS-82 & $<1$ \\
T687A; T690C; C696T; & & \\
T699C; G702A; T705C & KS-82/1 & 63 \\
\hline
\end{tabular}

Values for the spliced fraction of mRNA were determined as in Table 1 . Splicing activities relative to wild-type splicing are indicated.

whether folding of the intron was impaired in the stop codon mutants, CYT-18 was overexpressed together with the mutants, and in vivo splicing activity was tested (Fig. 6B). While CYT-18 has little effect on the wild-type activity, as observed previously (Mohr et al. 1992), splicing in the stop codon mutants was efficiently rescued. Rescue was efficient regardless of the position of the stop codon. However, splicing rescue by CYT-18 leads to an increase in aberrant products. Rescued splicing in the mutant KS-223, for example, results in $\mathrm{P}+8$, $P+9$, and increased $P+12$ in addition to $P+16$ products (Fig. 6B, Iane 2). M utants KS-27, KS-15, and KS-5 produce less cryptic $P+8$ product when rescued by CYT-18, consistent with the fact that the mutations destabilize the cryptic P1 stem (Figs. 6B, lanes 4,8,10, and Fig. 3C). These results are another indication, that in the absence of translation the pre-RNA is unable to fold into the splicing-competent structure.

Identification of an upstream sequence that interferes with splicing

When a stop codon is located 82 nucleotides upstream from the $5^{\prime}$ splice site, splicing activity is below $1 \%$ of wild type, while a stop codon located 27 nucleotides upstream allows a splicing activity of $10 \%$ (Table 1 ). This suggests that a sequence occurs between these two positions that negatively affects splicing. To narrow down and to identify such a sequence, two further mutants containing stop codons at positions -51 and -60 were constructed (Table 4). These two mutants spliced much better than KS-82. This result is indicative of a sequence between -60 and -80 that might interfere with folding of the intron, thereby inhibiting splicing. This idea is easily testable by altering the exon sequence in the $-60 /-80$ region. We constructed a mutant with a stop codon at position -82 with an al tered downstream sequence containing five point mutations spanning from position -79 to -64 in exon 1 . In case this region undergoes basepairing with intron sequences, it is expected that a potential long-range interaction would be disrupted by the point mutations. In the context of the five point mutations, the stop codon at position -82 should then no longer result in a total ly splicing-deficient construct. In- deed, splicing activity of this multiple mutant was found to be $26 \%$ (Table 4). This result is a strong evidence in support of the idea that this region interferes with splicing by base-pairing with intron sequences.

We subsequently searched for intron sequences complementary to the $-60 /-80$ region. We found a potential candidate located just upstream of the $3^{\prime}$ splice site. This potential interaction consists of 9 bp comprising nucleotides -81 to -73 of exon 1 and the terminal nucleotides of the intron (Fig. 7). This region is essential for the formation of the three dimensional structural elements P9.0a and P9.0b (Michel et al. 1989; Jaeger et al. 1993). Further experiments will be undertaken to verify this interaction, but compensatory mutations within the intron are problematic, as the splicing-competent structure must be maintained.

\section{Discussion}

It is generally assumed that group I intron splicing is facilitated by RNA-binding proteins in vivo. A specific in vivo splicing factor for the td intron has not yet been found, and many attemps to isolate splicing-defective mutants in trans were unsuccessful (R. Schroeder and $M$. Belfort, unpubl.). However, it was shown that the StpA protein as well as the ribosomal protein S12 can enhance splicing in vitro (Coetzee et al. 1994; Zhang et al. 1995). Both proteins bind RN A nonspecifically, suggesting an RN A chaperone activity (Herschlag 1995).

Splicing was uncoupled from translation in the group I intron containing $\mathrm{T} 4$ phage-derived td gene by introducing nonsense codons in the upstream exon of the premRN A. This resulted in severe splicing deficiency. The lack of spliced mRNA in the stop codon mutants is attributable neither due to nonsense-mediated mRNA decay nor to the invol vement of the translation product of exon 1 in splicing. A suppressor tRN $A_{U G A}$ partially rescues splicing in a UGA stop codon mutant. This is the first strong evidence that translation is required for efficient splicing in vivo. This evidence is further strengthened by the fact that translation inhibitors also lead to

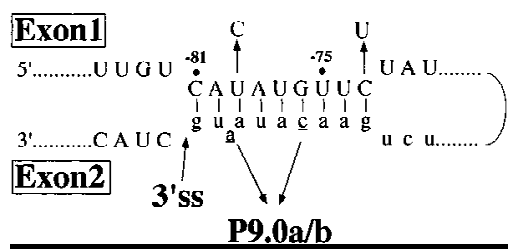

Figure 7. Long-range interaction between exon 1 and the $3^{\prime}$ terminus of the intron. Potential base-pairing interaction between exon positions -81 to -73 and $3^{\prime}$-terminal sequences of the intron. N egative positions indicate distance to the 5 ' splice site. Exon sequences are in uppercase, intron sequences in lowercase letters. (3' ss) 3' splice site. Mutations in mutant KS-82/ 1 , which disrupt this pairing, are indicated. Underlined lowercase letters indicate intron positions involved in the three dimensional elements P9.0a and P9.0b (Jaeger et al. 1993; Michel et al. 1989). 
splicing deficiency. Many years ago, Schmelzer and Schweyen observed that chloramphenicol repressed splicing of yeast mitochondrial introns and suggested not only that mitochondrial proteins were necessary but that the translation machinery might interact more directly with intron maturation (Schmelzer and Schweyen 1982).

There are two possibilities for how the ribosome can promote splicing: (1) by resolving exon-intron interactions that interfere with intron folding and (2) by direct stabilization of the splicing competent structure. We present experimental evidence for the first function, and several observations suggest that the second mechanism cannot be ruled out.

To test whether the splicing defect in the stop codon mutants results from the inability of the pre-RN A to fold into the splicing competent structure, splicing rescue was tried with two proteins that are involved in RNA folding, the ribosomal protein S12 and CYT-18. S12 has been shown previously to activate trans-splicing of the td intron in vitro, interacting with exon sequences rather than with intron sequences (Coetzee et al. 1994). In its natural context, the ribosome, S12 has been implicated in tuning translational accuracy by modulating the dissociation constants of tRN A s in the P- and A-sites of the small ribosomal subunit (Powers and Noller 1994; Karimi and Ehrenberg 1996). Overexpression of S12 partially rescues splicing of the stop codon mutants. We assume that this S12 activity comes from S12 protein nonassociated to the ribosome. One possible role for S12 in splicing of pre-RNA introns might be the destabilization of exon structures that interfere with intron folding.

A stop codon positioned at -82 reduced splicing activity below $1 \%$, whereas a nonsense codon positioned at -60 al lows $60 \%$ of wild-type splicing. This phenomenon could be attributable to the existence of exon sequences between these positions that interfere with intron sequences. In excellent agreement with this idea, additional point mutations in the -82 stop codon mutant rescue splicing from below $1 \%$ to $63 \%$ relative splicing activity. This is a strong evidence, that the $-82 /-60$ region interferes with splicing by undergoing base-pairing with intron sequences, disrupting the active intron conformation. In support of this idea, a sequence of 9 nucleotides, representing the $3^{\prime}$ terminus of the intron was found to be complementary to positions -81 to -73 .

Thus it is very likely that the main function of the ribosome in splicing is to "iron out" exon sequences and to resolve potential exon-intron interactions, which prevent the intron from folding into the splicing competent structure. However, as stop codons even close to the $5^{\prime}$ splice site (at position -5 and -15 ) affect splicing, we think that the ribosome does more than just resolve exon structures. The ribosome might also stabilize the intron structure in a more specific way, like CYT-18 does. When CYT-18 is overexpressed in the mutants containing stop codons in exon 1, spli cing is efficiently restored. Because CYT-18 helps to fold group I introns specifically, it is clear that the defect caused by the stop codons in exon 1 is a lack of correct fol ding. The ribosome might be able to recognize group I introns as they bear tRN Alike structural features (Caprara et al. 1996a,b). Preliminary binding assays with intron RNA to 705 ribosomes suggest that the ribosome can indeed bind the intron RN A with an affinity similar to mRN As (K. Semrad and R. Schroeder, unpubl.). For further and profound studies, binding-assay conditions have to be established, which resemble the actual splicing situation: an occupied P-site of the ribosome and an mRN A, which ends at the P-site, such that binding of the intron to an mRNA-vacant Asite can be measured. The ability of ribosomes to bind molecules of that size is demonstrated by binding of the 10Sa (tm) RNA to E. coli ribosomes (Tu et al. 1995; Keiler et al. 1996). The 10Sa RN A has tRN A and mRN A properties, suggesting that the ribosome can accommodate a quite diverse set of RNAs. Another very strong evidence that ribosomes can bind correctly fol ded group I intron RNA, comes from the observation that the Tetrahymena intron reverse splices into $50 \mathrm{~S}$ subunits and $70 S$ ribosomes (Roman and Woodson 1995).

There are several indications that the ribosome has additional functions, which are unrel ated to translation. Recently, it was shown that the ribosome is able to regulate the GTP-binding activity of a GTPase from the signal recognition particle (Bacher et al. 1996). Here, we present a first evidence for a novel type of ribosomal function: The ribosome seems to fine tune the folding of the intron RNA when splicing and translation are coupled.

\section{Materials and methods}

E. coli strains and growth media

E. coli strains used in this study were thymine-deficient derivatives of $\mathrm{C} 600$ strain $\left[\mathrm{F}^{-}\right.$, supE44, thi-1, thr-1, leuB6, lacY1, tonA 21 , thy ${ }^{-}$. M inimal media with and without thymine were as described (Belfort et al. 1983). For screening of the td phenotype, we used TBY-E +Thy and minimal medium. Bacto agar (1.5\%) was added for solid media. Antibiotics were used at the following concentrations: ampicillin (100 mg/liter), chloramphenicol (25 mg/liter), kanamycin (50 mg/liter), trimethoprim (25 mg/liter). Thymine was added at $50 \mathrm{mg} / \mathrm{liter}$.

\section{Plasmids}

All td constructs are in context of the vector pTZ18U (U.S. Biochemical ). The stop codon mutants in exon 1, the tdA770G/ UGA and the tdA771G/UAG mutants, the frameshift mutant (td-182 $/-18$ Ins), and the td $\Delta$ intron mutants with and without stop codon KS-82 were derived from in vitro mutagenesis (Kunkel et al. 1987). The mutant tdC870U was described previously (Schroeder et al. 1991). The vector pA 550 is a derivative of pACYC and carrying the trp tRN A synthetase from $\mathrm{N}$. crassa (Mohr et al. 1992). The vector pWKS $130 . S 12$ is a low-copy pACYC derivative and carries the ribosomal protein S12 under lac promoter (Coetzee et al. 1994; Wang and Kushner 1991). The su9 gene on the vector pJK 1 , al so compatible with the pT Z18U derivatives, codes for a suppressor tRN $A_{U G A}{ }^{T r p}$ (Kopelowitz et al. 1992).

\section{Oligonucleotides}

A list of mutagenic oligonucleotides follows (mutagenic resi- 
dues are underlined): KS-223; 5'-CCACTGTTTTCATTAAATTGGACC-3';

KS-82; 5'-ATAGAACATATGTCAAGGCGGTAAT-3'; KS-60; 5'-AAATAGCCATTACGTCAATTAAACTGATAGA-3'; KS-51; 5'-CAAATCCAAATATCAATTACGCACATT-3'; KS-27; 5'TCTACTGAGCGTCA $\overline{A T A C C A C T G C A-3 ' ; ~ K S-15 ; ~ 5 '-A C C C A-~}$ AGAAAACTTATACTGAGCGTTG-3';

KS-5/G781U; 5'-TCACCTTATACTAAGGCCTCAATTAACCCTAGAAAACATCTA-3'; td A770G/UGA; 5'-CTCAGGCCTCAATCAACCCAAGAAAAC-3'; td A771U/UAG; 5'-CTCAGGCCTCAACTAACCCAAGAAAAC-3'; td -182A; 5'-ATC AATAACTTCT $\triangle$ TAATTTGGTCTAC-3'; td -18 Ins; 5'-AAGAAAACATCTACCTGAGCGTTGAT-3'; tdA771U; 5'-ACTCAGGCCTCAAATAACCCAAGAAAAC-3'; td sintron; 5'GACGCAATATTAAACGGTAGACCCAAGAAAACATCTACTG-3'; KS-82/1; 5'-GCCATTACGCACATTGAATTGGTAAAACATGTGICAAGGCGGTAAT-3'. The oligo nucleotide for the primer-extension assay is NBS-2; 5'-GAGCAATATTAAACGGT-3'.

\section{In vivo RNA isolation}

For the isolation of cellular RNA the lysozyme freeze-thaw method was used as described (Belfort et al. 1990) with the following modifications: The cells were grown with IPTG added to a final concentration of $2 \mathrm{~mm}$. For the extraction protocol the RNA was precipitated without addition of $\mathrm{Mg}(\mathrm{OAC})_{2}$ but with $1 / 100$ volume of $0.5 \mathrm{M}$ EDTA instead.

For the time curve of mRNA degradation (Fig. 2), rifampicin was added at an $O D_{600}$ of 0.2 to a final concentration of 10 $\mu \mathrm{g} / \mathrm{ml}$ and samples were taken from time points between 0 and $40 \mathrm{~min}$. The following protocol was performed as described.

\section{Primer extension reaction to determine splicing of $t d$}

The primer extension assay and was described previously (M ohr et al. 1992, see also Fig. 1B) with the following modifications: The reactions were annealed in a $90^{\circ} \mathrm{C}$ water bath for $1 \mathrm{~min}$ with $10 \mu \mathrm{g}$ of total RNA (maximal volume of $2.5 \mu \mathrm{l}$ ), $1 \mathrm{ng}{ }^{32} \mathrm{P}$ labeled primer $(1 \mu \mathrm{l})$ and $1 \mu \mathrm{l}$ of hybridization buffer $(50 \mathrm{~mm}$ Tris- $\mathrm{HCl}$ at $\mathrm{pH} 8.4,60 \mathrm{~mm} \mathrm{KCl}$ ). The probes were allowed to cool to $45^{\circ} \mathrm{C}$. For the extension reaction $2.2 \mu \mathrm{l}$ of extension mix $\left[1 \mu \mathrm{l} \mathrm{H}_{2} \mathrm{O}, 0.6 \mu \mathrm{l}\right.$ of extension buffer $(500 \mathrm{~mm}$ Tris- $\mathrm{HCl}$ at $\mathrm{pH} 8.4$, $50 \mathrm{~mm} \mathrm{MgCl}, 50 \mathrm{~mm}$ DTT), $0.3 \mu \mathrm{l}$ poisoned mix (6 $\mu \mathrm{l} 10 \mathrm{~mm}$ dCTP, $6 \mu l 10 \mathrm{~mm}$ dATP, $6 \mu \mathrm{l} 10 \mathrm{~mm}$ dGTP, $25 \mu \mathrm{l} 10 \mathrm{~mm}$ ddTTP) and $0.3 \mu \mathrm{l} \mathrm{AMV}$ reverse transcriptase $(4 \mathrm{U} / \mu \mathrm{l})$ ] were added. The reaction was incubated at $42^{\circ} \mathrm{C}$ for $45 \mathrm{~min}$. After precipitation, formamide loading dye was added and half of the reaction was loaded on a $8 \%$ polyacrylamide gel (20: 1 acrylamide:bis-acrylamide, 7 м urea).

\section{In vitro transcription}

In vitro transcription was performed under suboptimal conditions to prevent hydrolysis of the transcripts: The td-containing plasmids were linearized with EcoRV. The transcriptions were performed in a total volume of $40 \mu \mathrm{l}$ with $40 \mathrm{~mm}$ Tris- $\mathrm{HCl}(\mathrm{pH}$ 7.4), $11 \mathrm{~mm} \mathrm{M} \mathrm{gCl}, 2 \mathrm{~mm}$ spermidine, $10 \mathrm{~mm} \mathrm{~N} \mathrm{aCl}, 2 \mu \mathrm{g}$ linearized template, $2.5 \mathrm{~mm}$ ATP, GTP, UTP and $0.5 \mathrm{~mm}$ CTP, 30 $\mu \mathrm{Ci}$ [35S] CTP, $25 \mathrm{~mm}$ DTT, 40 units of RN asin, and 20 units of T 7 polymerase. The transcriptions were incubated over night at $4^{\circ} \mathrm{C}$ and then isolated from a 5\% polyacrylamide gel (39:1 acrylamide:bis-acrylamide, $7 \mathrm{~m}$ urea) and eluted over night in $400 \mu$ l elution buffer $(250 \mathrm{~mm} \mathrm{Na}$ acetate, $0.1 \%$ SDS, $1 \mathrm{~mm}$
EDTA, $10 \mathrm{~mm}$ Tris- $\mathrm{HCl}$ ). After precipitation and resuspension, the transcripts were quantified in a scintillation counter.

\section{In vitro splicing}

In vitro transcripts (40 $000 \mathrm{cpm}$ ) were incubated for $3 \mathrm{~min}$ at $56^{\circ} \mathrm{C}$ with splicing buffer $(50 \mathrm{~mm}$ Tris- $\mathrm{HCl}$ at $\mathrm{pH} 7.3,5 \mathrm{~mm}$ $\mathrm{M} \mathrm{gCl}_{2}, 0.4 \mathrm{~mm}$ spermidine) in a total volume of $10 \mu \mathrm{l}$. The assay was incubated at room temperature for $5 \mathrm{~min}$, and then GTP was added to a final concentration of $10 \mu \mathrm{m}$. The reactions were performed at $37^{\circ} \mathrm{C}$, stopped at different time points by the addition of stop solution (10 mM EDTA, $5 \mu \mathrm{g}$ of tRN A) and precipitated. The reactions were resuspended in formamide dye and loaded on a 5\% polyacrylamide gel (39: 1 acrylamide:bisacrylamide, $7 \mathrm{~m}$ urea).

\section{Phosphorlmager quantification}

For the quantifications of the primer-extension products, the program Image Quant was used. The percentage of splicing was calculated with the formula: mRNA + crypticRNA)x100/ (preRN A +mRNA +crypticRN A. For mRN A splicing, the area of the mRNA product ( $P+16$ or $P+15$ and $P+7$, respectively) in addition with the reverse transcriptase stops at positions $\mathrm{P}+12$ and $\mathrm{P}+13$ were integrated plus the integrated area of cryptic splicing $(P+8)$ and compared to the integrated area of the preRNA $(P+5)$ by above mentioned formula. Values in Table 1 are from at least eight individual experiments, in Table 2 from at least two individual experiments, except for the mutant tdA771U, which was done only once.

\section{Acknowledgments}

We thank Hanna Engelberg-Kulka for the tRNA suppressor, $M$ arlene Belfort for the S12 plasmid and Alan Lambowitz for the CYT-18 plasmid. We especially thank Elisabeth Clodi for her input into this work. We thank U. von Ahsen, C. Behrens, M. Belfort, P. Brion, T. Coetzee, B. Cousineau, M. Cusick, I. Hoch, R. Lease, M. Parker, B. Streicher, S. Wallace, H. Wank, D. Wittberger, and S. Woodson for critical comments on the manuscript. This work was funded by the Austrian Science Foundation (FWF) grants nos. 9457 and 10615 to R.S.

The publication costs of this article were defrayed in part by payment of page charges. This article must therefore be hereby marked "advertisement" in accordance with 18 USC section 1734 solely to indicate this fact.

\section{References}

Bacher, G., H. Luetcke, B. Jungnickel, T.A. Rapoport, and B. Dobberstein. 1996. Regulation by the ribosome of the GTPase of the signal-recognition particle during protein targeting. Nature 381: 248-251.

Belfort, M., A. Moelleken, G.F. Maley, and F. Maley. 1983. Purification and properties of $\mathrm{T} 4$ thymidylate synthase produced by the cloned gene in an amplification vector. J. Biol. Chem. 258: 2045-2051.

Belfort, M., P.S. Chandry, and J. Pedersen-Lane. 1987. Genetic delineation of functional components of the group I intron in the phage T4 td gene. Cold Spring Harbor Symp. Quant. Biol. 52: 181-192.

Belfort, M., K. Ehrenman, and P.S. Chandry. 1990. Genetic and molecular analysis of RNA splicing in Escherichia coli. Methods Enzymol. 181: 521-539.

Caprara, M.G., V. Lehnert, and A.M. Lambowitz and E. West- 
hof. 1996a. A tyrosyl-tRNA synthetase recognizes a conserved tRN A-like structural motif in the group I intron catalytic core. Cell 87: 1135-1145.

Caprara, M.G., G. Mohr, and A.M. Lambowitz. 1996b. A tyrosyl-tRN A synthetase protein induces tertiary folding of the group I intron catalytic core. J. Mol. Biol. 257: 512-531.

Carreras, C.W. and D. Santi. 1995. The catalytic mechanism and structure of thymidylate synthase. Annu. Rev. Biochem. 64: 721-762.

Cech, T.R. 1990. Self-splicing group I introns. Annu. Rev. Biochem. 59: 543-568.

Cech, T.R., S.H. Damberger, and R. Gutell. 1994. Representation of the secondary and tertiary structure of group I introns. Nature Struct. Biol. 1: 273-280.

Chandry, P.S. and M. Belfort. 1987. Acitvation of a cryptic 5' splice site in the upstream exon of the phage T4 td transcript: Exon context, missplicing, and mRNA deletion in a fidelity mutant. Genes \& Dev. 1: 1028-1037.

Chu, E. and C.J. Allegra. 1996. The role of thymidylate synthase as an RNA binding protein. BioEssays 18: 191-198.

Chu, E., D. Voeller, D.M. Koeller, J.C. Drake, C.H. Takimoto, G.F. Maley, F. Maley, and C. Allegra. 1993. Identification of an RN A binding site for human thymidylate synthase. Proc. Natl. Acad. Sci. 90: 517-521.

Coetzee, T., D. Herschlag, and M. Belfort. 1994. Escherichia coli proteins, including ribosomal protein S12, facilitate in vitro splicing of phage T4 introns by acting as RNA chaperones. Genes \& Dev. 8: 1575-1588.

Gampel, A., M. Nishikimi, and A. Tzagoloff. 1989. CBP2 protein promotes in vitro excision of a yeast mitochondrial group I intron. Mol. Cell Biol. 9: 5424-5433.

Gott, J. M., A. Zeeh, D. Bell-Pederson, K. Ehrenman, M. Belfort, and D.A. Shub. 1988. Genes within genes: Independent expression of phage T4 intron open reading frames and the genes in which they reside. Genes \& Dev. 2: 1791-1799.

Herschlag, D. 1995. RN A chaperones and the RN A folding problem. J. Biol. Chem. 270: 20871-20874.

Herschlag, D., M. Khosla, Z. Tsuchihashi, and R.L. Karpel. 1994. An RN A chaperone activity of non-specific RN A binding proteins in hammerhead ribozyme catalysis. EMBO J. 13: 2913-2924.

Jaeger, L., Westhof, E., and Michel, F. 1993. Monitoring of the cooperative unfol ding of the sunY group I intron of bacteriophage T4. The active form of the sunY ribozyme is stabilized by multiple interactions with $3^{\prime}$ terminal intron components. J. Mol. Biol. 234: 331-346.

Karimi, R.Z. and M. Ehrenberg 1996. Dissociation rates of peptidyl-tRNA from the P-site of E. coli ribosomes. EMBO J. 15: 1149-1154.

Keiler, K.C., P.R.H. Waller, and R.T. Sauer. 1996. Role of a peptide tagging system in degradation of proteins synthesized from damaged messenger RNA. Science 271: 990-993.

Kopelowitz, J., C. Hampe, R. Goldman, M. Reches, and H. Engel berg-Kulka. 1992. Influence of codon context on UGA suppression and readthrough. J. Mol. Biol. 225: 261-269.

Kunkel, T.A., J.D. Roberts, and R.A. Zakour. 1987. Rapid and efficient site-specific mutagenesis without phenotype selection. Methods Enzymol. 154: 367-382.

Lambowitz, A.M. and M. Belfort. 1993. Introns as mobile genetic elements. Annu. Rev. Biochem. 62: 587-622.

Lambowitz, A.M. and P.S. Perlman. 1990. Involvement of aminoacyl-tRN A synthetases and other proteins in group I and group II intron splicing. Trends Biochem. Sci. 15: 440-444.

Lazowska, J.C., C. Jacq, and P.P. Slonimski. 1980. Sequence of introns and flanking exons in wild-type and box 3 mutants of cytochrom b reveals an interlaced splicing protein coded by an intron. Cell 22: 333-348.

Michel, F. and E. Westhof. 1990. M odelling of the three-dimensional architecture of group I catalytic introns based on comparative sequence analysis. J. Mol. Biol. 216: 585-610.

Michel, F., M. Hanna, R. Green, D.P. Bartel, and J.W. Szostak. 1989. The guanosine binding site of the Tetrahymena ribozyme. Nature 342: 391-395.

Mohr, G., A. Zhang, J.A. Gianelos, M. Belfort, and A.M. Lambowitz. 1992. The Neurospora CYT-18 protein suppresses defects in the Phage T 4 td intron by stabilizing the catalytically active structure of the intron core. Cell 69: 483-494.

Mohr, G., M.G. Caprara, Q. Guo, and A.M. Lambowitz. 1994. A tyrosyl-tRN A synthetase can function similarly to an RN A structure in the Tetrahymena ribozyme. Nature 370: 147150.

Murphy, F.L. and T.R. Cech. 1993. An independently folding domain of RNA tertiary structure within the Tetrahymena ribozyme. Biochemistry 32: 5291-5300.

N ilsson, G., J.G. Bel asco, S.N. Cohen, and A. von Gabain. 1987. Effects of premature termination of translation on mRNA stability depends on the site of ribosome rel ease. Proc. Natl. Acad. Sci. 84: 4890-4894.

Öhman-Heden, M., A. A hgren-Stal handske, S. Hahne and B.M. Sjöberg. 1993. Translation across the $5^{\prime}$-splice site interferes with autocatalytic splicing. Mol. Microbiol. 7: 975-982.

Powers, T. and H.F. Noller. 1994. Selective perturbation of G530 of $16 \mathrm{~S}$ rRN A by translational miscoding agents and a streptomycin-dependence mutation in protein S12. J. Mol. Biol. 235: 156-172.

Roman, J. and S.A. Woodson. 1995. Reverse splicing of the Tetrahymena IVS: Evidence for multiple reaction sites in the 23S rRN A. RNA 1: 478-490.

Schmelzer, C. and R.J. Schweyen. 1982. Evidence for ribosomes involved in splicing of yeast mitochondrial transcripts. Nucleic Acids Res. 10: 513-524.

Schroeder, R., U. von Ahsen, and M. Belfort. 1991. Effects of mutations of the bulged nucleotide in the conserved $\mathrm{P} 7$ pairing el ement of the phage $\mathrm{T} 4 \mathrm{td}$ intron on ribozyme function. Biochemistry 30: 3295-3303.

Tsuchihashi, Z., M. Khosla, and D. Herschlag. 1993. Protein enhancement of hammerhead ribozyme catalysis. Science 262: 99-102.

Tu, G.F., G.E. Reid, J.G. Zhang, R.L. Moritz, and R.J. Simpson. 1995. C-terminal extension of truncated recombinant proteins in Escherichia coli with a 10Sa RNA decapeptide. J. Biol. Chem. 270: 9322-9326.

Voeller, D.M., L.-M. Changchien, G. Maley, F. Maley, T. Takechi, R.E.W.M. Turner, C.J. Allegra, and E. Chu. 1995. Characterization of a specific interaction between Escherichia coli thymidylate synthase and Escherichia coli thymidylate synthase mRN A. Nucleic Acids Res. 25: 869-875.

von Ahsen, U., J. Davies, and R. Schroeder. 1991. Antibiotic inhibition of group I ribozyme function. Nature 353: 368370.

- - . 1992. N on-competitive inhibition of group I intron RN A self-splicing by aminoglycoside antibiotics. J. Mol. Biol. 226: 935-941.

Walstrum, S.A. and O.C. Uhlenbeck. 1990. The self-splicing RNA of Tetrahymena is trapped in a less active conformation by gel purification. Biochemistry 29: 10573-10576.

Wang, R.F. and S.R. Kushner. 1991. Construction of versatile low-copy number vectors for cloning, sequencing and gene expression in Escherichia coli. Gene 100: 195-199.

Waring, R.B., P. Towner, S.J. Minter, and R.W. Davies. 1986. 
Splice-site selection by a self-splicing RN A of T etrahymena. Nature 321: 133-139.

Weeks, K.M. and T.R. Cech. 1995a. Efficient protein-facilitated splicing of the yeast mitochondrial bl 5 intron. Biochemistry 34: 7728-7738.

- - - 1995b. Protein facilitation of group I splicing by assembly of the catalytic core and the $5^{\prime}$ splice site domain. Cell 82: 221-230.

Woodson, S.A. 1992. Exon sequences distant from the splice junction are required for efficient self-splicing of the Tetrahymena IVS. Nucleic Acids Res. 20: 4027-4032.

Woodson, S.A. and T.R. Cech 1991. Alternative secondary structures in the $5^{\prime}$ exon affect both forward and reverse self-splicing of the Tetrahymena intervening sequence RN A. Biochemistry 30: 2042-2050.

Woodson, S.A. and V.L. Emerick. 1993. An alternative helix in the $26 \mathrm{~S}$ rRN A promotes excision and integration of the Tetrahymena intervening sequence. Mol. Cell. Biol. 13: 11371145.

Zaug, A.J., M.M. McEvoy, and T.R. Cech. 1993. Self-splicing of the group I intron from Anabaena pretRN A: Requirement for base-pairing of the exons in the anticodon stem. Biochemistry 32: 7946-7953.

Zhang, A., V. Derbyshire, J.L. Galloway Salvo, and M. Belfort. 1995. Escherichia coli protein StpA stimulates self-splicing by promoting RNA assembly in vitro. RNA 1: 783-793. 


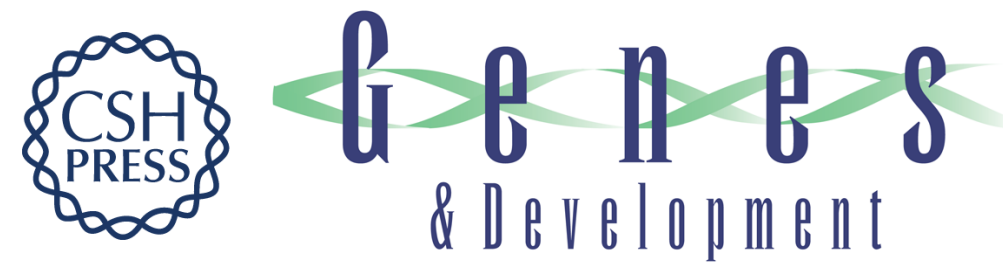

\section{A ribosomal function is necessary for efficient splicing of the T4 phage thymidylate synthase intron in vivo}

Katharina Semrad and Renée Schroeder

Genes Dev. 1998, 12:

References This article cites 51 articles, 15 of which can be accessed free at:

http://genesdev.cshlp.org/content/12/9/1327.full.html\#ref-list-1

License

Email Alerting Receive free email alerts when new articles cite this article - sign up in the box at the top Service right corner of the article or click here.

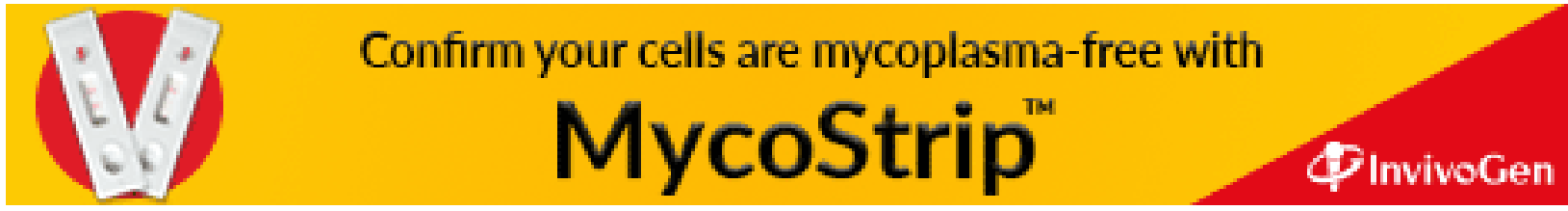

